\title{
Alunos com TDAH (Transtorno de Déficit de Atenção e Hiperatividade): um desafio na sala de aula
}

\author{
Students with ADHD (Attention Deficit Hyperactivity Disorder): a challenge in the class room
}

Alumnos con TDAH (Transtorno de Déficit de Atención y Hiperactividad): un desafío en el salon de clase

Luciana Teles Moura ${ }^{1}$, Katiane Pedrosa Mirandola Silva ${ }^{2 *}$, Keliene Pedrosa Mirandola Silva².

\section{RESUMO}

Objetivo: Revisar na literatura científica as dificuldades encontradas pelos professores de alunos com diagnóstico de Transtorno de Déficit de Atenção e Hiperatividade (TDAH) no processo de ensino. Métodos: Trata-se de uma pesquisa de abordagem qualitativa do tipo de revisão de literatura buscando responder a seguinte problemática: Quais as principais dificuldades enfrentadas pelos professores de alunos com TDAH no processo de ensino-aprendizagem? Resultados e Discussão: O Transtorno de Déficit de Atenção e Hiperatividade (TDAH), é caracterizado pela presença de um desempenho inapropriado nos mecanismos que regulam a atenção, os impulsos e as atividades motoras e que podem afetar diretamente o rendimento escolar de alunos com esse transtorno. A partir dessa pesquisa bibliográfica foi possível conhecer esse transtorno e alguns desafios que os professores enfrentam com alunos diagnosticados com TDAH no processo de ensinoaprendizagem em sala de aula. Considerações finais: Compreender o TDAH é essencial para que a escola tenha uma visão sistêmica do processo ensino-aprendizagem em que esse aluno está inserido, para contribuir no pleno desenvolvimento da aprendizagem dos TDAHs, além da inclusão e aceitação dos mesmos por toda comunidade escolar.

Palavras-chave: TDAH, Alunos, Desafio, Aprendizagem, Escola.

\begin{abstract}
Objective: Review, in scientific literature, the difficulties encountered by teachers of students with a diagnosis of Attention Deficit Hyperactivity Disorder (ADHD) in the teaching process. Methods: A qualitative research with the intention to accomplish with literature review the answer to the following problems: What are the main difficulties faced by teachers of students with ADHD in the teaching-learning process? Results and Discussion: Attention Deficit Hyperactivity Disorder (ADHD) is characterized by the presence of inappropriate performance in the mechanisms that regulate attention, impulses and motor activities and that can directly affect the school performance of students with this disorder. From this bibliographic research it was possible to learn about this disorder and some challenges that teachers face with students diagnosed with ADHD in the teaching-learning process in the classroom. Final considerations: Understanding ADHD is essential for the school to have a systemic vision of the teaching-learning process in which that student is even, to contribute to the full development of ADHD learning, in addition to the inclusion and acceptance of them by the school community.
\end{abstract}

Keywords: ADHD, Students, Challenge, Learning, School.

1 Doutorado em Psicologia pela Universidade Federal do Espírito Santo, Brasil.

${ }^{2}$ Mestrado em Gestão Social, Educação e Desenvolvimento Regional pela Faculdade Vale do Cricaré - ES, Brasil. E-mail: katianepms@yahoo.com.br 


\section{RESUMEN}

Objetivo: Revirar, en la literatura científica las dificultad enfrentadas por los profesores de alumnos que tiene diagnóstico de Trastorno de Déficit de Atención y Hiperactividad (TDAH) en el proceso de enseñanza. Métodos: Se trata de una investigación de abordaje cualitativo del tipo de revisión de literatura buscando responder a la siguiente problemática: ¿Cuáles son las principales dificultades enfrentadas por los profesores de alumnos con TDAH en el proceso de enseñanza-aprendizaje Resultados y Discusión: El Trastorno de déficit de Atención y Hiperactividad (TDAH), se caracteriza por la presencia de un desempeño inapropiado en los mecanismos que regulan la atención, los impulsos y las actividades motoras y que pueden afectar directamente el rendimiento escolar de alumnos con ese trastorno. A partir de esa investigación bibliográfica fue posible conocer ese trastorno y algunos desafíos que los profesores enfrentan con alumnos diagnosticados con TDAH en el proceso de enseñanza-aprendizaje en el aula. Consideraciones finales: Comprender el TDAH es esencial para que la escuela tenga una visión sistémica del proceso enseñanzaaprendizaje en que ese alumno está incluso, para contribuir en el pleno desarrollo del aprendizaje de los TDAHs, además de la inclusión y aceptación de los mismos por toda comunidad escolar.

Palabras claves: TDAH, Estudiantes, Desafío, Aprendizagem, Escolar.

\section{INTRODUÇÃO}

Os transtornos de aprendizagem estão presentes no âmbito escolar, tornando-se desafios para a escola que, por sua vez, precisa garantir um ensino de qualidade a todos os alunos. De acordo com o Código Internacional de Doenças (CID-10, 1993), os transtornos nos quais os padrões normais de aquisição de habilidades são perturbados desde os estágios iniciais do desenvolvimento, não são simplesmente uma consequência de uma inexistência de oportunidade de aprender nem são derivados de qualquer forma de traumatismo ou de doença cerebral adquirida, ao contrário, pensa-se que os transtornos resultam de anormalidades no processo cognitivo, que derivam em grande parte de algum tipo de disfunção biológica.

Diante desse contexto, os transtornos de aprendizagem têm sido um desafio constante para os profissionais que estão preocupados com a garantia de ensino eficaz a todos os alunos. Trata-se de um ramo extensivamente explorado pela neurociência e pela psicopedagogia, e pouco explorado no âmbito da pedagogia, e os pedagogos são ou deveriam ser os principais interessados no tema, já que são os que mais convivem com essa clientela na escola e precisam buscar mais conhecimentos sobre o que e quais são os distúrbios, transtornos ou problemas que influenciam a aprendizagem, quais as principais peculiaridades e como lidar com eles (BOIMARE S, 2011).

Dentre muitos transtornos de aprendizagem, neste artigo destaca o TDAH, no qual predomina a desatenção e/ou hiperatividade, e que pode afetar diretamente a aprendizagem do aluno no âmbito escolar. O Transtorno de Déficit de Atenção e Hiperatividade (TDAH) tem causado preocupações aos professores em sala de aula, pois os alunos com esse diagnóstico podem apresentar dificuldades de aprendizagem, visto que esse transtorno nasce do que se chama trio de base alterada que é formado por alterações de atenção, da impulsividade e da velocidade da atividade física e mental (SILVA ABB, 2003).

O TDAH é um transtorno do desenvolvimento do autocontrole que consiste em problemas com os períodos de atenção, o controle do impulso e o nível de atividade, sendo assim a criança com esse transtorno apresenta dificuldades de controlar as emoções e o próprio comportamento (BARKLEY R, 2002).

Mediante essas dificuldades que os alunos com TDAH apresentam, surge a surge a seguinte problemática: Quais as principais dificuldades enfrentadas pelos professores de alunos com TDAH no processo de ensinoaprendizagem?

Os docentes se tornam pontos importantes de apoio para os alunos com TDAH, visto que ao utilizarem novas estratégias e se comprometerem com o ensino, consequentemente, contribuem para a elevação da autoestima e da satisfação desses alunos perante as próprias conquistas. 


\section{MÉTODOS}

A presente pesquisa de abordagem qualitativa, trata-se de uma revisão da literatura, a metodologia utilizada seguiu os princípios da pesquisa bibliográfica visando agrupar e analisar estudos científicos. Determinou-se primordialmente a pesquisa em meios eletrônicos, gratuitos e de acesso público para análise de fontes secundárias tais como livros didáticos, guias e manuais com a temática em questão.

A busca de materiais e o acesso ocorreu entre agosto a outubro de 2018. Os descritores utilizados foram: TDAH na escola, alunos com TDAH e as dificuldades na sala de aula, o desafio dos professores com os alunos com TDAH.

A contribuição dos autores dos estudos analíticos no material pesquisado favoreceu a descrição dos desafios encontrados pelos professores de alunos com TDAH em sala de aula que impulsionam estes profissionais a buscarem novas estratégias de ensino.

\section{RESULTADOS E DISCUSSÃO}

Para Rohde LA e Benczik EB (1999), o TDAH é um problema de saúde mental determinado por desatenção, agitação ou hiperatividade e impulsividade, podendo acarretar dificuldades emocionais, familiares, sociais e escolares. Na escola, os portadores desse transtorno podem apresentar dificuldades de aprendizagem na sala de aula, bem como relacionamentos conturbados em outros ambientes sociais.

\section{Fatores causais do TDAH}

O TDAH é uma das síndromes neuropsiquiátricas consideradas mais comuns, que se configura pela presença de alguns sintomas relacionados entre si, os quais podem manifestar-se ou não simultaneamente; a frequência e a gravidade da desatenção, hiperatividade ou impulsividade de uma criança devem ter caráter surpreendente quando comparadas a de outras da mesma idade (DINIZ NO e SENA SS, 2007).

Muitas são os possíveis fatores causais do TDAH, as evidências mais fortes que o Distúrbio do Déficit de Atenção apresentam uma alteração na estrutura cerebral de seus portadores, vêm de numerosos estudos realizados por meio de exames de neuroimagem, esses estudos retratam uma hipoperfusão cerebral, localizada mais significativamente na região pré-frontal e pré-motora do cérebro, isto é, significa que a região frontal, nas pessoas com TDAH, recebe um menor aporte sanguíneo (SILVA ABB, 2014).

Conforme discorre Silva ABB (2003), a ação reguladora do comportamento humano é feita pelo lado frontal, que exerce uma série de funções de caráter inibitório, cabendo a ele 'puxar o freio de mão' do cérebro humano no que diz respeito aos seus pensamentos, impulsos e velocidade de suas atividades físicas e mentais.

Rohde LA e Benczik EB (1999), articulam que o TDAH tem ligação com a hereditariedade, ou seja, estudos realizados em famílias de crianças e adolescentes com TDAH, como gêmeos, têm indicado significativa participação de um componente genético na gênese do transtorno. Para Barkley $R$ (2002), a nicotina e o álcool, quando consumidos durante a gravidez, podem causar variações em algumas partes do cérebro do bebê, incluindo a região frontal orbital, e mulheres que tiveram complicações no parto causando sofrimento fetal, podem ter mais chance de terem filhos com TDAH.

Outro possível fator que pode ter como consequência o TDAH refere-se aos problemas familiares. Ainda de acordo com a linha de pensamento de Rohde LA e Benczik EB (1999), um estilo parental muito permissivo pode ser consequência e não causa do transtorno, características familiares tais como: funcionamento familiar 
caótico, alto grau de discórdia conjugal; baixa instrução materna; famílias com nível socioeconômico mais baixo; famílias com apenas um dos pais ou responsáveis, ou que o pai abandona a família.

De tal modo, observa-se que é primordial buscar constantemente conhecer mais acerca desse transtorno, tanto por parte dos profissionais da educação quanto da família, desde o diagnóstico até o tratamento, de forma a contribuir para oferecer, no âmbito escolar, um ensino significativo para os TDAHs.

\section{Tipos de TDAH}

Segundo Diniz NO e Sena SS (2007) a dificuldade de dispensar atenção a detalhes ou errar por descuido em atividade escolares e de trabalho, dificuldade para manter a atenção em tarefas ou atividades lúdicas, parece não ouvir quando Ihe direciona a palavra; não seguir regras e não terminar tarefas escolares, domésticas ou deveres profissionais; dificuldades em organizar tarefas escolares, domésticas ou deveres profissionais; perder coisas necessárias para tarefas ou atividades e ser facilmente entretido por estímulos alheios à tarefa e demonstrar esquecimento em atividades diárias, são características de aluno com TDAH.

O TDAH é um transtorno que se apresenta de três formas, sendo conhecido por Transtorno de Déficit de Atenção com Hiperatividade, denominado TDAH. No entanto, para Phelan TW (2005), o TDA, sem o H, referese aos predominantes desatentos, com dificuldades de prestar atenção e são mais comuns em meninas. Já o TDAH, predominantemente hiperativo ou impulsivo, revela-se mais presente em meninos, é marcado pela agitação e problemas de comportamento que geram na escola. De acordo com o APA (1980), há também o tipo combinado com similiar em desatenção e hiperatividade. Antes era usado o termo TDA, aplicado no DSM III (Manual Estatístico das Doenças Mentais, 3ª edição), sendo substituído no DSM - IV para TDAH, sempre determinando predomínio de desatenção ou hiperatividade e impulsividade ou ainda o tipo combinado (APA, 1994).

De acordo com Oliver L (2011), O DDA, Desordem de Déficit de Atenção, é uma condição semelhante ao TDAH, com as mesmas peculiaridades, mas que pode ser tratado com uma mediação pedagógica ou psicopedagógica. O professor poderá ajudar efetivamente os TDAHs caso se disponha a utilizar estratégias que facilitarão o cotidiano escolar desses estudantes, estimulando a criatividade, aumentando a autoestima e contribuindo para o desenvolvimento integral deles em sala de aula.

\section{O TDAH e as dificuldades na sala de aula}

O aluno com TDAH provoca uma reflexão constante na atuação pedagógica, adaptações do ensino ao estilo de cada aprendiz, atendendo as necessidades educacionais individuais (ROHDE LA, BENCZIK EBP, 2003). O professor é desafiado a refletir em suas estratégias de ensino pelos alunos com TDAH, e devem buscar adaptações que facilitarão a aprendizagem desses alunos que necessitam de um atendimento especializado e diferenciado.

Compreende-se que a maioria dos educadores encontram dificuldades em lidar com alunos portadores de transtornos, inclusive com o TDAH. Por isso, é importante que a escola dê suporte para que seus professores aperfeiçoem seus conhecimentos e habilidades (CARVALHO RE, 2000), com 0 intuito de desvendar 0 significado da essência do aprender para os alunos TDAHs.

A intervenção psicopedagógica também é imprescindível, uma vez que existem lacunas de aprendizagem que precisam de um trabalho de restruturação das habilidades e conteúdo, e o acompanhamento pedagógico em conjunto com o professor previne essas lacunas na aprendizagem. Esses profissionais também propiciam aos pais informações que auxiliam na administração do comportamento destes alunos TDAHs em casa, contribuindo para a melhoria de suas habilidades educacionais e sociais. Procurar ajuda médica e/ou terapêutica tornará a vida desses estudantes mais produtiva, já que eles estarão ao seu lado no árduo trabalho de construção e reconstrução de sua vida (SILVA ABB, 2009).

REAS/EJCH | Vol. Sup. 22 | e611 | DOI: https://doi.org/10.25248/reas.e611.2019 Página 4 de $\mathbf{7}$ 
De acordo com informações disponíveis no portal da ABDA (2018), o tratamento do TDAH deve ser uma conjunção de medicamentos, instrução aos pais e professores, além de técnicas exclusivas ao portador.

Nota-se diferentes direções na evolução do TDAH em função do ambiente familiar e social, visto que quanto mais organizada e adequada for a estrutura familiar, melhor será a evolução possível do quadro TDAH. Diversas pesquisas indicam que ambientes sociais caóticos e adversos estão fortemente relacionados com o agravamento do quadro e com o surgimento simultâneo de outros transtornos (DINIZ NO e SENA SS, 2007). Mediante isso, percebe-se que a família poderá contribuir para amenizar as dificuldades causadas por esse transtornos na vida dos alunos com TDAH.

O TDAH costuma se evidenciar ainda na infância e, em cerca de $70 \%$ dos casos, o transtorno prossegue na vida adulta, com isso, deve-se enfatizar que o comportamento dos TDAHs precisa ser compreendido e bem dirigido por eles próprios e por todos que os cercam desde os primeiros anos escolares, contribuindo, assim, para uma vida adulta mais feliz (SILVA ABB, 2014).

Conforme descrevem Dupaul GJ e Stoner G (2007): crianças com TDAH, com frequência "perturbam" o desenvolvimento das atividades em sala de aula e, portanto, atrapalham a aprendizagem dos colegas. $O$ acolhimento da escola com os TDAHs deve constituir uma relação empática e pôr em prática atitudes como ouvir, refletir e demonstrar afeto por estes alunos, considerando que todos necessitam se sentir bem acolhidos no espaço escolar.

Diante desse cenário, vale ressaltar que a criança com TDAH se torna um desafio para o professor, pois a mesma causa agitação na sala de aula, e o educador precisa manter o equilíbrio nas aulas para garantir uma aprendizagem a todos os alunos, alguns professores sentem tanta frustração quanto aos pais desses alunos, pois também eles são seres humanos únicos, com características específicas e estilos de ensino particular, e nenhum conjunto isolado de orientações e estratégias funciona na inter-relação de todos os professores com todos os alunos, surge então a necessidade de adequar os métodos a cada aluno, inclusive ao portador de TDAH (BENCZIK EBP, BROMBERG MC, 2003).

Muitas são as dificuldades encontradas em sala de aula pelos professores no processo de ensinoaprendizagem, conforme citado por vários autores até aqui. Mas, quando se trata de alunos com transtorno de déficit de atenção e hiperatividades, essas dificuldades podem até causar frustração. De acordo com Albano AMS et al. (2012), os TDAHs, desenvolvem uma agitação motora significativa e permanente: não consegue ficar sentada na carteira, não permanece em silêncio, tenta finalizar as atividades propostas no menor tempo possível, não aguenta esperar, mexe ininterruptamente pés, mãos, demonstra dificuldade em lidar com o "não", etc.

Silva ABB (2009) também apresenta algumas características do comportamento da criança com TDAH no âmbito escolar: mexe ou sacode pés e mãos regularmente e é naturalmente distraída por estímulos externos. Isto é, essas crianças não conseguem ficar quietas e concentradas na aula, qualquer barulho ou movimento as impede de fixar sua atenção no que o professor diz.

Assim, para a autora, o TDAH também apresenta dificuldades de esperar sua vez em brincadeiras ou em situações de grupo, em seguir instruções e ordens, problemas de relacionamento com seus colegas, geralmente são pessoas teimosas, e isto é perceptível nas brincadeiras ou situações em grupo. A dispersão também é comum na vida dos TDAH, pois querem fazer tudo ao mesmo tempo e não conseguem concluir nada, tendo em vista as dificuldades em perpetuar a atenção em tarefas, atividades lúdicas e a frequência de mudar de uma atividade inacabada para outra (SILVA ABB, 2009).

E a autora complementa o raciocínio ao afirmar que o TDAH tem dificuldade de ser objetivo ao falar e

REAS/EJCH | Vol. Sup. 22 | e611 | DOI: https://doi.org/10.25248/reas.e611.2019 Página $\mathbf{5}$ de $\mathbf{7}$ 
esquece, frequentemente, de fazer algo solicitado por outra pessoa, às vezes fala excessivamente e vive perdendo itens necessários para tarefas ou atividades escolares (SILVA ABB, 2009). Devido ao TDAH, percebe-se que os alunos com esse transtono enfrentam muitas dificuldades no processo de ensinoaprendizagem, daí a importância da escola intervir positivamente no processo de aprendizagem desses alunos.

O estresse provocado por ambientes desestruturados, ou mesmo o aumento de demandas na atuação pessoal ou social, podem intensificar em grande escala os sintomas do TDAH, deve então adequar ambientes escolares para atender as especificações dos alunos com TDAH a fim de auxiliá-los no desempenho de suas atividades na sala de aula, pois, sabe-se que esses estudantes já possuem as próprias dificuldades de desatenção e até mesmo de hiperatividade, o que dificulta o desempenho pessoal e social (SILVA ABB, 2009). A escola deve evitar situações que venham intensificar os sintomas desse transtorno, propiciando ambientes bem mais estruturados e adaptados de acordo com as necessidades desses alunos.

Sabe-se que as escolas regulares buscam promover sempre a organização de seus alunos a fim de somar adaptações que possibilitem valorizar as potencialidades dos alunos de forma inclusiva, pois a inclusão constitui um grande desafio as escolas regulares, que estão sendo chamadas para levar em conta a diversidade e as características e necessidades dos alunos, empregando um modelo nele centrado com ênfase na aprendizagem (CARVALHO RE, 2000).

Visto que as dificuldades na sala de aula são desafios encontrados pela escola com vários alunos, notase que é preciso buscar vencer esses desafios a partir de estratégias que visem atender as necessidades de cada um com ênfase na aprendizagem, inclusive os diagnosticados com TDAH.

\section{CONSIDERAÇÕES FINAIS}

Pode-se concluir que o TDAH é um transtorno que afeta o aluno, causa dificuldades na aprendizagem, gera insegurança e ansiedade. É possível responsabilizar esse transtorno pelos comportamentos apresentados pelos estudantes no desenvolvimento cognitivo e intelectual. Foi possível constatar que os alunos com TDAH podem apresentar dificuldades no processo de ensino-aprendizagem em sala de aula, que podem ser vencidas quando o professor se propõe a confrontar os próprios saberes, atuações e práticas com novos conhecimentos e estratégias, visando um ensino de qualidade. A forma de ensinar um aluno TDAH demonstrou-se complexa e difícil, portanto é importante que se perceba o potencial que esse aluno poderá ou não desenvolver, e que tenha capacidade de reconhecer a responsabilidade dele sobre o resultado. $O$ professor deve ter um equilíbrio emocional, ser criativo, e conhecer com propriedade o transtorno do aluno, pois, assim, terá mais facilidade para obter a participação desses educandos em sala de aula. Cabe a escola propiciar aos alunos com Transtorno de Déficit de Atenção e Hiperatividade, aprendizagens significativas de acordo com suas potencialidades, procurando garantir a todos um ensino de qualidade e de forma inclusiva.

\section{REFERÊNCIAS}

1. ABDA, Associação Brasileira do Déficit de Atenção. Manual diagnóstico e estatístico dos transtornos mentais V, 2018.

2. ALBANO AMS, VICENZI E, et al. A criança com TDAH: metodologias e adaptações curriculares. Curitiba: Fael, 2012.

3. APA, American Psychiatric Association. Diagnostic and statistical manual of mental disorders. (3rd ed.). Washington: American Psychiatric Association, 1980.

4. APA, American Psychiatric Association. Diagnostic and statistical manual of mental disorders: DSM-IV. 4. Ed. Washington, DC: APA, 1994.

5. BARKLEY R. Transtorno de déficit de atenção/hiperatividade (TDAH): guia completo e autorizado para pais, professores e profissionais da saúde. Porto Alegre: Artmed, 2002. 
6. BENCZIK EBP, BROMBERG MC. (2003). Intervenções na escola. Em L. A. Rohde \& P. Mattos (Orgs.), Princípios e práticas em transtorno de déficit de atenção/hiperatividade (p. 199-218). Porto Alegre: Artmed, 2003.

7. BOIMARE S. Crianças impedidas de pensar. São Paulo: Editora Paulinas, 2011.

8. CARVALHO RE. Removendo Barreiras para a Aprendizagem. Educação Inclusiva. Porto Alegre: Artes Médicas, 2000.

9. CID-10. Classificação de Transtornos Mentais e de Comportamento da CID-10: Descrições clínicas e diretrizes diagnósticas. Organização Mundial de Saúde (Org.). Porto Alegre: Artes Médicas, 1993.

10. DINIZ NO, SENA SS. Distraído e a 1000 por hora: guia para familiares, educadores e portadores de transtorno de déficit de atenção/hiperatividade. Porto Alegre: Artmed, 2007.

11. DUPAUL GJ, STONER G.TDAH nas Escolas. São Paulo: M. Books do Brasil, 2007.

12. OLIVER L. Distúrbios de aprendizagem e de comportamento: Como detectar, entender e tratar os problemas de aprendizagem. Um guia indispensável para pais, professores e profissionais da saúde. Rio de janeiro: Wak Editora, 2011.

13. PHELAN TW. TDA/TDAH: Transtorno de déficit de atenção e hiperatividade. São Paulo: M Books do Brasil Editora Ltda., 2005.

14. ROHDE LA, BENCZIK EBP. Transtorno de Déficit de Atenção/Hiperatividade. O que é? Como posso ajudar? Porto Alegre: Artes Médicas; 1999.

15. ROHDE LA, BENCZIK EBP. Transtorno de Déficit de Atenção/ Hiperatividade. O que é? Como posso ajudar? Porto Alegre: ARTMED, 2003.

16. SILVA ABB. Mentes Inquietas. Entendendo Melhor o Mundo das Pessoas distraídas, Impulsivas e Hiperativas. São Paulo: Gente, 2003.

17. SILVA ABB. Mentes inquietas: TDAH; desatenção, hiperatividade e impulsividade. 4. Ed. Rio de Janeiro: Objetiva, 2009.

18. SILVA ABB. Mentes inquietas: TDAH; desatenção, hiperatividade e impulsividade. Ana Beatriz Barbosa Silva. 4. Ed. - São Paulo: Globo, 2014. 